\title{
Collaborative Web-Based Experimentation in Flexible Engineering Education
}

\author{
Denis Gillet, Anh Vu Nguyen Ngoc, and Yassin Rekik
}

\begin{abstract}
The Ecole Polytechnique Fédérale de Lausanne (EPFL), Lausanne, Switzerland, is deploying a flexible learning scheme for selected pilot courses in engineering education. In such a scheme, traditional lectures and written exercises are combined with additional Web-based learning resources. The main objective of this initiative is to sustain the evolution from traditional teaching to active learning and to better integrate the increasing number of educational resources available online. In engineering education, a key activity to sustain the learning process is hands-on experimentation carried out using either simulation tools or real equipment. This paper describes how a collaborative Web-based experimentation environment has been introduced at the EPFL for providing more flexibility to students performing laboratory experiments in automatic control, biomechanics, and fluid mechanics. It particularly describes the eJournal, a Web service integrated in the proposed learning environment that enables the collection and sharing of preparatory notes and experimental results with both peers and teaching assistants.
\end{abstract}

Index Terms-Automatic control, collaborative learning, engineering education, flexible learning, Internet, remote experimentation, Web-based instruction.

\section{INTRODUCTION}

$\mathbf{T}$ HE EMERGING knowledge society poses challenges to traditional academic institutions in many aspects, including pedagogical, technological, and organizational ones. An integrated approach toward a flexible education paradigm has to be taken to ensure the necessary evolution for coping with this ineluctable, and hopefully valuable, mutation of society. The reason for such a paradigm shift is because of the students' needs in terms of professional competencies and personal development, including the necessary skills for teamwork. The motivation for flexible education at the level of the academic institutions is mainly a question of competitiveness in attracting students and in positioning as centers of excellence.

From a pedagogical perspective, flexible education means providing students with extended accessibility to learning resources, increased freedom to organize their learning activities, and enhanced participation, autonomy, and collaboration. From a technological perspective, flexible education corresponds to an adequate exploitation of the information and communica-

\footnotetext{
Manuscript received August 2, 2004; revised May 20, 2005. Elements presented in this paper result from various e-learning projects and activities ${ }^{1}$ supported in part by the Board of the Swiss Federal Institutes of Technology and of the European Union under the fifth and sixth framework programs. ${ }^{2}$

The authors are with the Ecole Polytechnique Fédérale de Lausanne (EPFL), CH-1015 Lausanne, Switzerland (e-mail: denis.gillet@epfl.ch).

Digital Object Identifier 10.1109/TE.2005.852592

${ }^{1}$ http://emersion.epfl.ch

${ }^{2} \mathrm{http} / / / \mathrm{www}$.prolearn-project.org
}

tion devices and infrastructures, especially the Internet. From an organizational point of view, flexible education relies on renewed study programs, regulations, as well as partnerships and collaborations with other institutions. Trying to deploy flexible education in such perspectives means less teaching and more learning opportunities [1], which rely on additional resources, alternative modalities, and personalized support offered in an open academic environment.

Flexible learning can be seen as an extended version of blended or integrated learning in which the students can choose, to some extent, the resources and the modalities. In such a flexible framework, the students only select the resources and the modalities that provide a real added value, which is quite challenging from an authoring and educational prospective.

In engineering education, the transition toward a flexible education scheme [2] necessitates as a first step a flexible access to experimentation resources [3]-[7] and to collaboration facilities [8]. The collaboration facilities sustain both the collaborative learning among students and the online tutoring offered by teaching assistants (TAs) to students. Collaboration plays a very important role in knowledge building, sharing, and distribution, especially in a flexible context where students can follow different learning modalities to perform multisession experiments. The literature seems to support that collaboration among learners has a significant impact on learning outcomes [9]-[11].

One should emphasize that Web-based experimentation is not only a means to carry out practical learning activities in engineering education but also a matter subject to study. Remote monitoring, testing, and control are becoming increasingly important in manufacturing, process control, and customer support.

This paper presents the solutions deployed and the approach chosen by the School of Engineering at the Ecole Polytechnique Fédérale de Lausanne (EPFL), Lausanne, Switzerland, to provide students with more flexibility by offering collaborative Web-based experimentation modules. Section II of the paper briefly presents the learning setting and the Web-based learning environment. Section III details the eJournal, the main component introduced to sustain collaborative Web-based experimentation. In Section IV, the notion of continuity of interaction is presented. Section V details the evaluation methodology and results. The paper ends with some concluding remarks and perspectives.

\section{WEB-BASED EXPERIMENTATION}

\section{A. The Learning Settings}

In engineering education, the practical activities are as important as the theoretical ones. In the spirit of flexible learning, 
students have the possibility of performing an experiment at any time and from any location, thus benefiting from a more effective cognitive experience. In other words, the student is provided with the opportunity to follow different learning modalities. The modalities vary according to the presence of teaching assistants (TAs) and according to the student's location. When group members work together in the presence of TAs, they are in a face-to-face ( $\mathrm{f} 2 \mathrm{f}$ ) condition. The location can be a laboratory with full access to physical equipment and instrumentation (local condition), a computer room on the campus (near condition), a student's home, or any other place (remote condition). In the last two conditions, students remotely access the physical laboratory equipment and/or computer simulation tools. Students can perform multisession experiments, i.e., they can do the first part of an experiment on campus and pursue the rest of it at home.

The academic year at the EPFL is divided into a winter and a summer term. Web-based experimentation has been progressively introduced since the 2000 winter term in automatic control, biomechanics, and fluid mechanics. However, only the hands-on activities in automatic control have been evaluated and are described here for conciseness.

These mandatory laboratory assignments in automatic control are offered to students from microengineering, electrical engineering, and mechanical engineering during the last year of the bachelor program. They are proposed concurrently with 56 lecture hours on digital control (analysis and design).

The laboratory assignments in automatic control are structured in three parts: introduction, experimentation, and examination. The introduction part is split into two modules dedicated to the presentation of the learning objectives, the learning modalities, and the learning tools. Special emphasis is given to the freedom offered by the flexible learning approach and the associated responsibilities in managing personal learning. The experimentation part is split into three to seven hands-on modules that correspond to the core learning activities. The number of requested hands-on modules depends on the different degree programs in which the students are enrolled. The examination part is carried out as a laboratory test.

The hands-on modules are composed of two parts. The first part is dedicated to a preliminary analysis and design activity called the prelab. When carrying out the prelab activities, the students can only access the virtual version of the laboratory experiments. Students have to submit a prelab document to a TA to be granted with the right to proceed further with the actual experimentation, called the labwork. The labwork consists of carrying out a real experiment and validating the preliminary design on the physical device. The prelab assignment has been introduced to ensure that students have the prior knowledge necessary to benefit from the hands-on experiment and to motivate them to do preparatory work on their own. No fixed schedule is imposed on the students; only the sequence of modules has to be followed. The laboratory test consists of performing a randomly selected, two-hour, hands-on experimentation module and then presenting the associated results for about 20 minutes to the professor in charge of the course. The course lasts for 14 weeks.

\section{B. The Web-Based Learning Environment}

A comparative study has been carried out to determine the most common features required for completing typical experimentation assignments by students enrolled in the third and fourth year of the engineering curricula at the EPFL. The Web-based learning environment resulting from this comparative study and from student observations contains all the components necessary to complete laboratory assignments successfully. Those components that are integrated in the so-called eMersion environment are heterogeneous in the sense that they are developed using different technologies and may be located on different servers.

The cockpit-like eMersion environment is a service-based environment [12] that enables the observation and manipulation of real laboratory systems, regardless of the chosen modality (local, near, or remote). In automatic control, the exploration carried out using this environment focuses on experimentation related to the understanding of the dynamic behavior of mechatronic systems and the practice of the different stages that must be completed in the design cycle of a digital controller. The environment is mainly dedicated to sustaining knowledge reinforcement and know-how acquisition rather than content delivery.

The environment includes three main parts: the experimentation console (Fig. 1), the toolkit console (Fig. 2), and the laboratory journal (Fig. 3). The experimentation console is an interactive Java applet that enables the actual realization of experiments, as the result of a remote access to real online equipment. The equipment is visualized in real time using a Web cam.

The applet shown in Fig. 1 is dedicated to the observation and the control of an electrical drive available at the EPFL. Its user interface enables the user to carry out an experiment on the remote equipment or to run a local simulation. Access to the physical equipment is only possible once the TA has validated the prelab. This interface allows the user in just one click to save measurements, snapshots of the built-in oscilloscope, and configuration parameters, in the laboratory journal. Typical tasks carried out by the students using this applet include the open-loop identification of the transfer function and the close-loop control of either the position or the velocity of the electrical drive using a proportional-integral-derivative (PID) controller that can be adjusted during the experiment. The available function generator can be used to generate either an excitation or a reference signal. Access to experiments offered by other institutions is also possible using the eMersion environment, providing the dedicated applets are available.

The laboratory journal, also called the eJournal, constitutes the asynchronous edition and collaboration part that facilitates reporting, knowledge integration, and sharing. The eJournal will be discussed in detail in the next section.

The toolkit console is an application based on the PHP scripting language, namely SysQuake Remote, which provides the necessary functionalities for preparing experiments and analyzing results. Fig. 2 shows a module interface of SysQuake Remote, where students can analyze their experimental data stored in the eJournal or loaded from the local disk, identify a nonparametric frequency response, and match the coefficients of a continuous-time transfer function. 


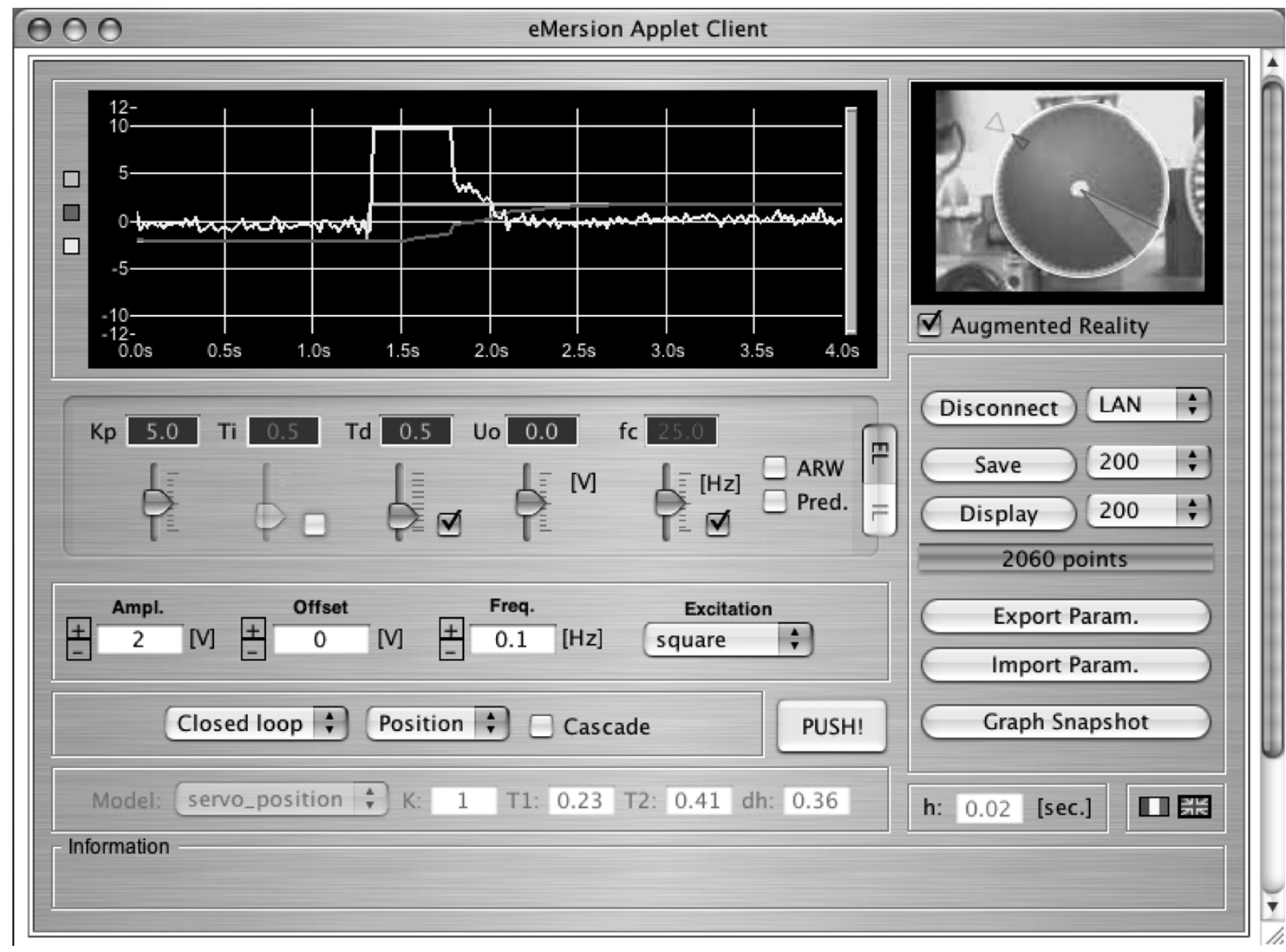

Fig. 1. User interface of the experimentation console integrated in the eMersion environment.

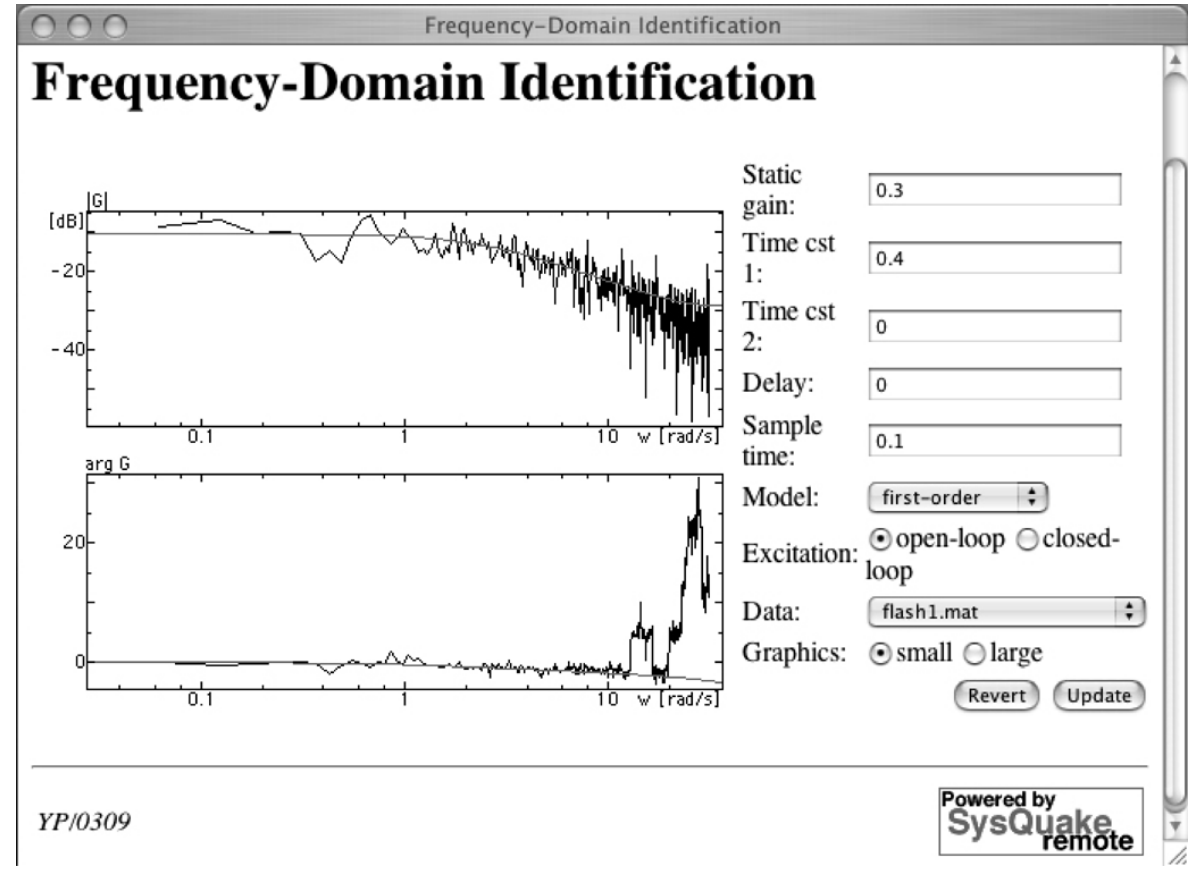

Fig. 2. Analysis panel of the toolkit console integrated in the eMersion environment.

The eMersion environment also provides access to supplementary information, such as experimentation protocol and related theory reminders; it shows the experimentation objective and the current task description.
III. THE eJOURNAL COLLABORATION WORKSPACE

The laboratory journal metaphor is introduced as a dedicated component of the Web-based learning environment to support 


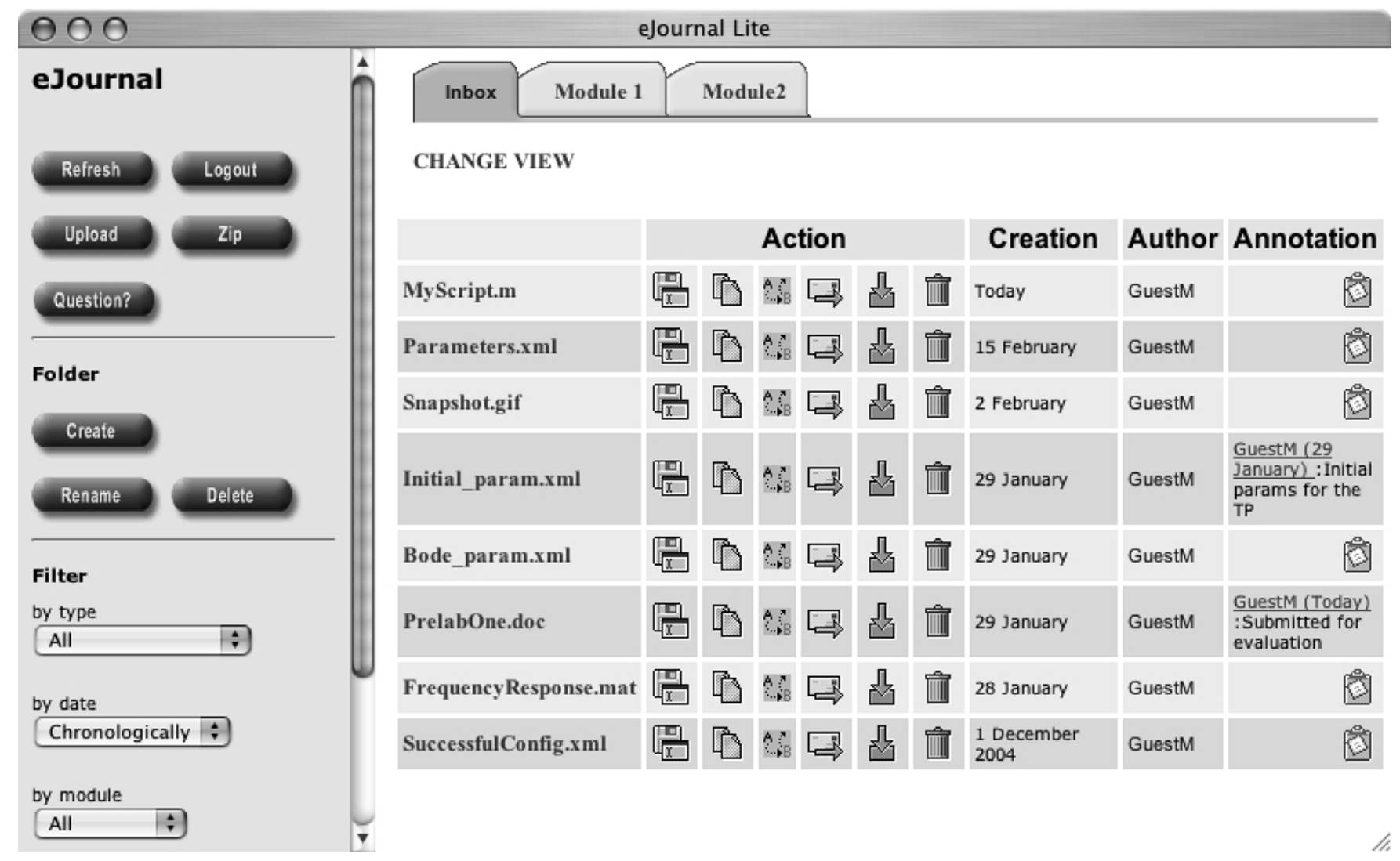

Fig. 3. eJournal collaboration space integrated in the eMersion environment.

the collaborative activities within groups of students committed to complete together a specific experimentation assignment. The laboratory journal metaphor was chosen since engineering students are familiar with this concept, and it is easy for them to use and conceptualize.

Electronic journals for personal use have much in common with paper journals. Like them, they serve as a work diary. However, the electronic content is easier to search, to browse, and to copy; thus, electronic journals encourage further exploitation of their content. Laboratory journals take a privileged place in research and engineering practices [13]. They serve as chronological repositories for experimentation planning, realization, and performance evaluation [14]. The benefit of taking electronic notes in science and engineering are even greater when the journal is shared among a group of co-workers. Hence, it seems also appropriate to use it in engineering education where such scientific methodology has to be acquired as well.

The eJournal, which plays the role of an asynchronous collaboration artifact, has been designed as an extension of the electronic journal paradigm that facilitates the hands-on and collaborative activities in a flexible learning context. The eJournal main space looks like the mailbox of an e-mail client, except that it does not contain e-mail but rich-type documents (Fig. 3).

The eJournal provides a common workspace, where students can store, retrieve, share, and exchange the group documents when preparing and performing the experiments. The documents stored in the eJournal are called fragments. All fragments are typed and represent different kinds of data. The fragments are categorized based on their sources and their content. For example, those uploaded from local disks are typed "uploaded fragment." Those generated from SysQuake Remote are typed "SQR." For those imported from other components, many different types are defined, such as "snapshot," which represents graphical data; "parameter set," which are sets of parameters [in forms of extended markup language (XML) data] imported from the manipulation applet; or "result," which are experimental results. Fragments with different types are handled differently. The fragments can be grouped into different folders. The fragments available in the workspace can be filtered based on different categories. They can be deleted temporarily (put to trash) or permanently (physically deleted). The temporarily deleted fragments can be restored. Tags can be assigned to fragments when they are created to ease their processing and sharing. A list of tags corresponding to the assigned tasks is automatically generated from the experimentation protocol and proposed in the save dialogue of the various Web components.

Using the eJournal and its fragments, students are provided with many different ways to collaborate with one another, with professors, and with TAs. Students can submit their fragments to the professor handling the summative evaluation or to the TAs handling the formative one. The fragments can be annotated. Students can directly send fragments with associated annotations or send questions with attached fragments to other groups or to TAs via an integrated e-mail system. This mechanism is used for prelab submission, and it can be used to get contextualized support. The eJournal also provides the possibility to copy/move fragments between eJournals belonging to different groups of students. In fact, students usually work in pairs, which 

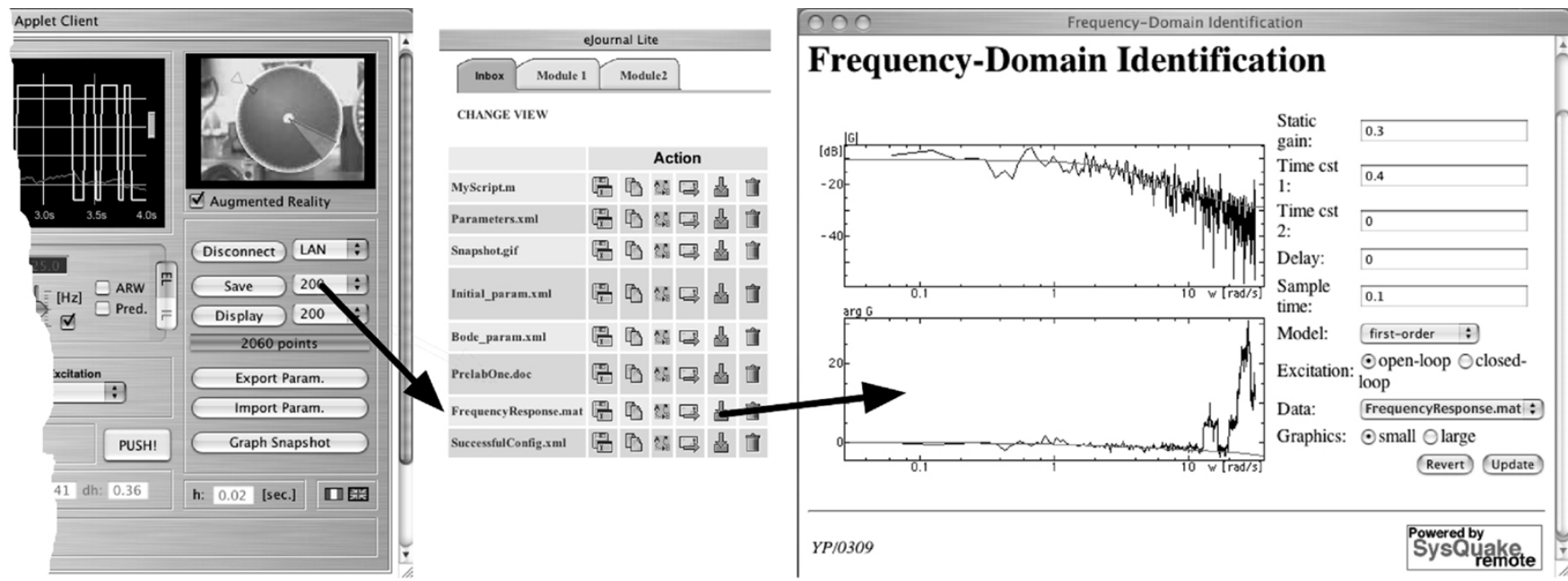

Fig. 4. eJournal and the continuity of interaction.

are formed by the students themselves; each pair owns a single eJournal.

The eJournal enables many services that generate awareness based on the fragment analysis and calculation. The awareness plays an important role in facilitating the teaching and collaborative learning processes, especially in a flexible context such as this one. Besides the availability awareness, such as the user presence (who is currently connected to the environment) and the user location (local, near, or remote condition), many other kinds of group awareness based on the fragment activities, called fragment-based awareness [8], are also provided.

\section{CONTINUITY OF INTERACTION}

The eJournal is a convenient medium to sustain the continuity of interaction across learning modalities, among members of the learning community and among Web components [15]. One can use the eJournal to upload fragments from local disks and also to import or export snapshots, parameter sets, and/or numerical results from or to other eMersion components, such as the experimentation applet or the toolkit component. This feature is important in the sense that the eJournal, as a laboratory journal, should support the experimental data input-output (I/O).

The concept of continuity of interaction means that interruptions of actions are avoided as much as possible, which is an important factor in the context of flexible hands-on experimentation. Fig. 4 represents an example of continuous interaction. The experimentation applet exports experimental results to the eJournal, which are then processed using the toolkit component. The operation is single-click driven. For example, a student working on campus can click on the "Export Results" button in the experimentation console to save the experimental result as a fragment typed "Results" in the group eJournal. Later at home, the same student or another member of the group can click on this fragment in the eJournal to automatically load the toolkit component. In fact, depending on the type of fragment, the appropriate component is launched. The continuous interaction mechanism eases the hands-on experimentation and collaboration activities, while reducing the cognitive overload that is typically observed when students use learning environments.
Data are passed smoothly and naturally from one component to another and from one modality to another. The requirement to use external applications for data sharing and exchanging is minimized. Users work with minimum discontinuity in all dimensions of interaction.

\section{Evaluation}

\section{A. Methodology}

The purpose of the evaluation was twofold; first, to find out to what extent the students were embracing the flexible learning paradigm (modalities) and, second, to assess the actual added values of the Web-based environment features and the quality of its user interface (usability). Thorough analysis of the fragments stored in the eJournal database and a questionnaire completed by students have been adopted as the evaluation approach. A few interviews with no statistical value have also been carried out for the purpose of getting a better understanding of the quantitative evaluation results. Since the eMersion environment was already in a production stage (actually used by regular students), the evaluation approach is more a summative process than a formative process. An ad hoc evaluation instrument, therefore, had to be chosen.

The instrument for the evaluation of the modalities relies on the fragment $\log$ analysis. Information available includes the creator, type, and creation date of the fragments, and the Internet protocol (IP) number of the client computers. This latest information enables the determination of the modality (local, near, or remote). Fragments that originate from components of the Web-based environment and that are directly imported to the eJournal are called intra fragments. Conversely, fragments that are uploaded from the local user's computer are called extra fragments. These are mostly created using stand-alone applications (no part of the eMersion environment). Fragments that are created in a face-to-face modality (local) are called $f 2 f$ fragments, while fragments created during near or remote learning modalities are called flexible fragments. These definitions of fragment categories allow the observation of two dimensions of the use of the environment. The first dimension is the amount of 


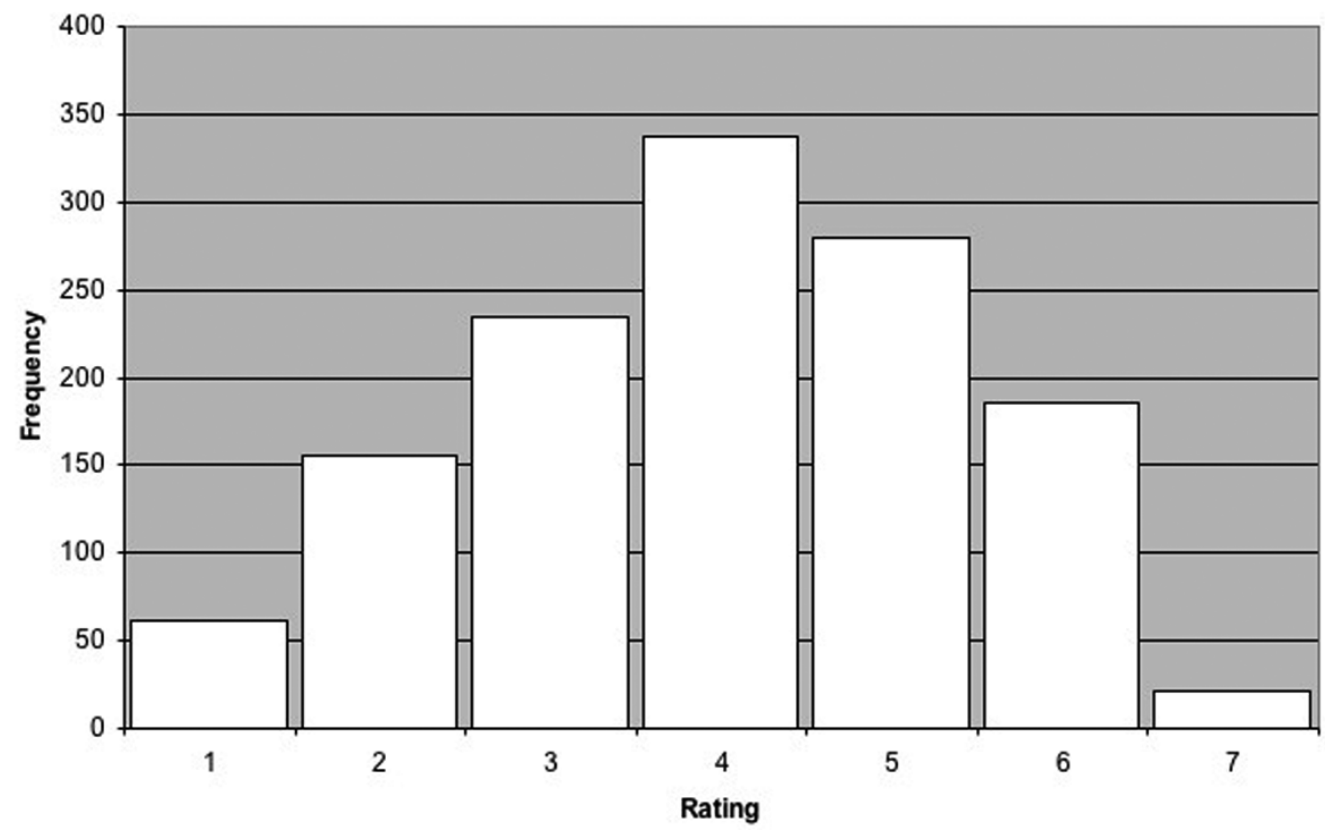

Fig. 5. Overall satisfaction rating for all questions.

the student's work that takes place within the Web-based environment compared with work that occurs outside. It is measured as an intra fragment ratio that is the number of intra fragments divided by the total number of fragments. This measure reflects the completeness of the environment for performing hands-on experimentation tasks. The second dimension is linked to the importance of f2f learning modalities compared with flexible learning modalities. It is quantified as the flexible fragment ratio that is the number of flexible fragments divided by the total number of fragments.

The instrument for the evaluation of the usability [16] relies on a Likert scale questionnaire [17], which contains 19 usability related assertions to which the student has to agree or disagree on a seven-point scale, ranging from 1 (strongly disagree) to 7 (strongly agree). These questions allow measuring the user satisfaction with computer system usability. This questionnaire has been extended with supplementary factual questions (such as name, sex, electronic courses taken, familiarity with computers, questions about the student's own computer if he or she has any, and perceived eJournal use). The names of the students have been requested to enable a neutral evaluator to correlate the log analysis and the questionnaires.

\section{B. Results}

The evaluation was carried out during the 2003 summer term with the 96 students enrolled in the microengineering degree program. The automatic control laboratory is a mandatory course for these students.

The 71 questionnaires (74\%) returned at the end of the term gave the following results. The mean of satisfaction was around 4.03 over $7(\mathrm{SD}=1.17)$. The statement "The system provides error messages that clearly help me to resolve problems" received the worst ranking with a mean of satisfaction of 2.74 $(\mathrm{SD}=1.47)$. This shows that the usability of the environment could be greatly improved by adjusting help and error messages. In fact, since implementing a help system is quite time consuming, only basic features were provided. Hence, this poor score was not a surprise. The responses to all the questions were combined, and the overall frequencies for each point of the scale were plotted (Fig. 5).

The supplementary factual questions have provided useful information about the background of the students, including the availability of an Internet connection at home $(83 \%)$ and the operating system they were using $(2.8 \%$ used Linux; $4.8 \%$ used Mac OS; $63 \%$ used Windows; $11.2 \%$ used both Linux and Windows; and the rest gave no information). Because of the very small female representation in the sample (only 4 out of 71), no gender differentiations were attempted.

Students were also asked to provide the three most positive and three most negative aspects (in order of importance) at the end of the questionnaire concerning the usage of the environment and the environment itself. The most frequent positive comment of the system was its flexibility ( 34 out of 85 of all positive comments referred to this aspect). Students considered that the environment had provided them with an excellent choice to perform their hands-on activities at any time and from any location. The integration of all the necessary tools in one integrated environment also appears to be important in the students' positive comments. Students also enjoyed different interactive and collaborative features provided by the eJournal. They also liked the hands-on activities that reinforced their theoretical knowledge.

The majority of negative comments (from a total of 71 negative comments) concerned technical problems (server and client crashes), the complexity of the interface (many windows for many tools), and again no provision for satisfactory help and error messages. The technical problems were twofold. A virus killed the Windows server handling the eJournal and the eMersion environment just before an $\mathrm{f} 2 \mathrm{f}$ session attended by many 


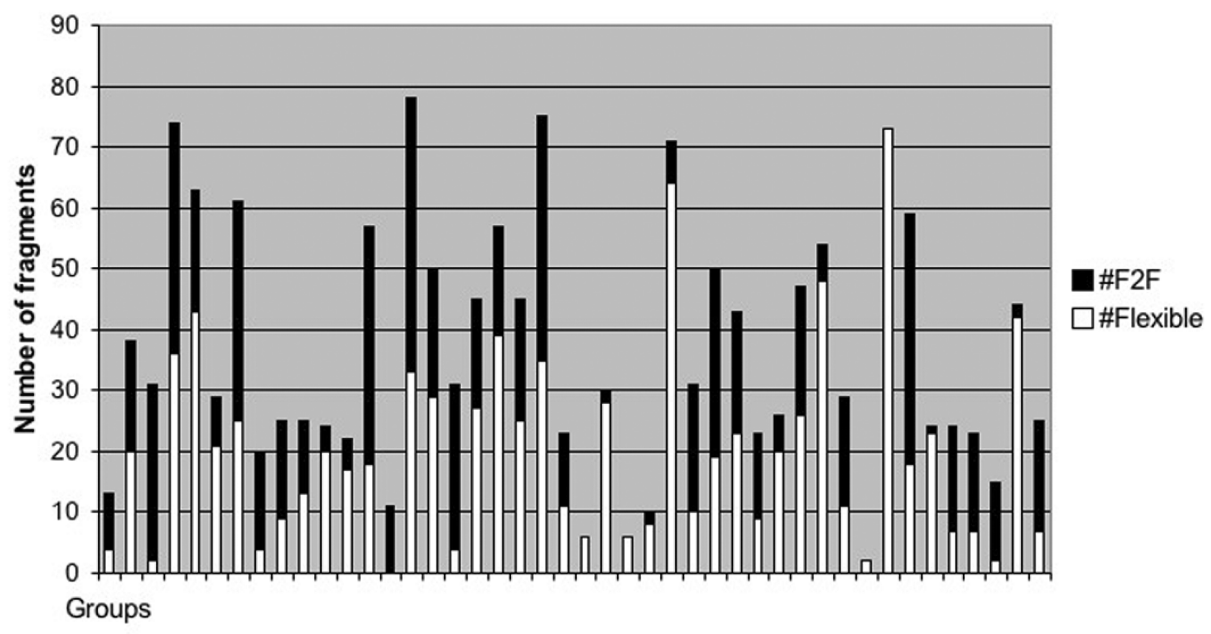

Fig. 6. f2f flexible fragments created during the 2003 summer term.

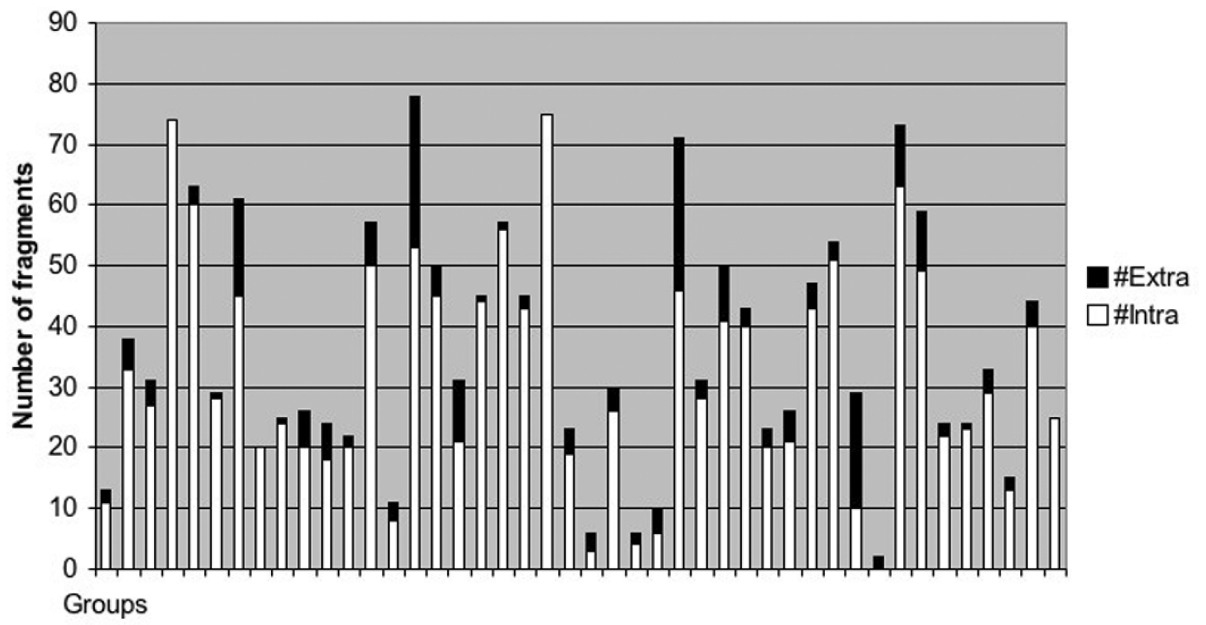

Fig. 7. Intra and extra fragments created during the 2003 summer term.

students. The Mac OS 9 computers available within the laboratory premises also experienced a few crashes. These equipment failures underline that remote experimentation is a quite complex setting in terms of information technologies deployment. The computers running the real-time controller of the laboratory experiments also manage the data and parameters exchange over the Internet. Moreover, in $\mathrm{f} 2 \mathrm{f}$ sessions, the students sit in front of the same computers to access through the Web the eMersion environment.

The fragment log analysis gave information on 44 active groups of two students. Four groups created no fragment at all. The active groups created a mean of 36 fragments in performing the practical modules (three mandatory hands-on modules) for the whole term. Eighty-six percent of the fragments were created within the environment with the experimentation component and the toolkit component; the other $14 \%$ were fragments created with stand-alone applications. The number of fragments created in flexible sessions corresponds to 55\%. The ratio of $\mathrm{f} 2 \mathrm{f}$ and flexible fragments shows that the students took advantage of the different learning modalities (Fig. 6). The ratio of the intra and extra fragments (Fig. 7) supports the assumption that the Web-based environment does not disturb students in conducting flexible hands-on experimentation compared with the traditional one. Thus, the environment usability has been found to be satisfactory.

Concerning other variables, besides the user acceptance and satisfaction that contributed to the participation assessment, the group performance (via the grade of the group members) has also been considered. However, no statistical correlation between the number of created fragments and the group performance was found for the term considered here.

\section{CONCLUDING REMARKS}

Collaborative Web-based experimentation is deployed at the Ecole Polytechnique Fédérale de Lausanne (EPFL), Lausanne, Switzerland, within pilot courses. It is particularly deployed on a large scale (about 160 students a year) for hands-on laboratory courses in automatic control education. It has proven to be an efficient means of promoting active learning within a flexible learning scheme. The eMersion environment deployed to sustain these initiatives can be used equally well in local, near, or remote conditions, thus providing a real choice of learning modalities. 
The eMersion environment has been designed to comply simultaneously with the needs of three different engineering courses using either simulation tools or real equipment. Hence, it is general enough to support different learning scenarios and to integrate different Web components developed for different courses by different institutions.

Collaborative Web-based experimentation successfully supports from a pedagogical point of view autonomous and collaborative learning activities. It also supports from a logistical point of view the handling of larger classes in a context where financial means to equip laboratory premises is limited. It finally enables the sharing of laboratory resources among partner universities. This last benefit has not yet been exploited to its full potential for technical compatibility issues, but research is being conducted to ease the integration of heterogeneous and distributed learning components [18].

The main contribution of this paper is a journal tool designed as an online work repository and a collaborative workspace, which is combined with the core experimentation component of the proposed Web-based learning environment. This tool, called the eJournal, is found to be the key added value that is suited to and appreciated by students. The eJournal via the Internet supports individual and collaborative activities within groups of students that are committed to complete together a specific experimentation assignment. It also supports group awareness and evaluation by keeping logs of the actions performed by the students.

The eJournal's main feature is to sustain the continuity of interaction at two levels. It enables and augments at a first level the asynchronous interaction process among the learning partners (students, professors, and teaching assistants). It serves as a shared workspace where students can maintain and share data in order to collaborate as well as document their practical work. It includes many advanced functionalities for sustaining the learning and collaboration process in flexible hands-on activities. It also allows educators to supervise students' progress and provide asynchronous support. At a second level, the eJournal enables the interaction between heterogeneous Web-based components, thus facilitating experimentation and reducing the cognitive overload even in local conditions. Finally, the eJournal enables the construction of different fragment-based metrics to evaluate individual and collaborative activities.

In the considered flexible learning context, the attempt to introduce synchronous collaboration tools, such as chat facilities, were not successful. The main reason is that students regularly meet on campus and, consequently, can interact directly when needed. In addition, they are usually more comfortable discussing equations and graphics $f 2 f$ rather than using electronic tools. If the students needed to interact when they were not on campus, they preferred to use general-purpose asynchronous communication solutions, such as SMS (short message service) or e-mail, than a tool dedicated to a single course.

The deployment of the collaborative Web-based experimentation environment detailed in this paper relied on an iterative development approach carried out during more than three years. During this period, user feedbacks and observations were taken into account to improve the successive versions of the environment and to fulfill the students' needs better.
Currently, a new version of the environment is under development. It will be released as an open-source software package to ease the sharing of resources within the academic community. This extended version supports the WebDAV protocol [19] for the storage of fragments on any file system. It also provides more possibilities for users to manage shared fragments collaboratively.

\section{ACKNOWLEDGMENT}

The authors would like to thank Dr. S. Sire for his essential contribution to the eJournal design and assessment.

\section{REFERENCES}

[1] D. Starr, "Virtual education: Current practices and future directions," Internet Higher Educ., vol. 1, no. 2, pp. 157-165, 1998.

[2] D. Gillet et al., "Toward flexible learning in engineering education," in Innovations 2003: World Innovations in Engineering Education and Research, W. Aung et al., Eds. Arlington, VA: iNEER and Begell, 2003 , pp. $95-102$.

[3] C. Schmid, "The virtual control lab VCLab for education on the web," in Proc. Amer. Control Conf., vol. 2, 1998, pp. 1314-1318.

[4] Y. Piguet and D. Gillet, "Java-based remote experimentation for control algorithms prototyping," in Proc. Amer. Control Conf., 1999, pp. 1465-1469.

[5] D. Magin et al., "Engineering students' understanding of the role of experimentation," Eur. J. Eng. Educ., vol. 25, no. 4, pp. 351-358, 2000.

[6] H. Saliah-Hassane, P. D. Burnett, and C. Loizeau, "RVLabX, A Webbased interactive laboratory environment for education and research," in Proc. 31st Annu. Frontiers in Education Conf., vol. 2, Oct. 2001, pp. T4C-5-T4C-9.

[7] R. B. Sepe and N. Short, "Web-based virtual engineering laboratory (VE-LAB) for collaborative experimentation on a hybrid electric vehicle starter/alternator," IEEE Trans. Ind. Appl., vol. 36, no. 4, pp. 1143-1150, Jul.-Aug. 2000.

[8] A. V. Nguyen, D. Gillet, and S. Sire, "Sustaining collaboration within a learning community in flexible engineering education," presented at the ED-MEDIA Conf., Lugano, Switzerland, Jun. 21-26, 2004. (Outstanding Paper Award).

[9] D. Jonassen et al., "Constructivism and computer-mediated communication in distance education," Amer. J. Distance Educ., vol. 9, no. 2, pp 7-26, 1995.

[10] D. Eastmond and J. Ziegahn, "Instructional design for the online classroom," in Computer Mediated Communication and the Online Classroom. Cresskill, NJ: Hampton Press, 1995, vol. 3, Distance Learning, pp. 29-36.

[11] Z. L. Berge and M. P. Collins, "Computer mediated communication and the online classroom," in Computer Mediated Communication and the Online Classroom. Cresskill, NJ: Hampton Press, 1995, vol. 1, Overview and Perspectives, pp. 1-10.

[12] D. Gillet et al., "The cockpit: An effective metaphor for Web-based experimentation in engineering education," Int. J. Eng. Educ., vol. 19, no. 3, pp. 389-397, 2003.

[13] J. B. McCormack et al., "The complementary roles of the laboratory notebooks and laboratory reports," IEEE Trans. Educ., vol. 34, no. 1, pp. 133-137, Feb. 1991.

[14] L. Leifer, "Design team performance: Metrics and impact of technology," in Evaluating Corporate Training: Models and Issues. Norwell, MA: Kluwer, 1997.

[15] A. V. Nguyen, D. Gillet, Y. Rekik, and S. Sire, "Sustaining the continuity of interaction in Web-based experimentation for engineering education," in Proc. Computer-Aided Learning in Engineering Education Conf. (CALIE) 2004, Grenoble, France, 2004, pp. 99-110.

[16] M. B. Rosson and J. M. Carroll, Usability Engineering Scenario-Based Development of Human-Computer Interaction. San Mateo, CA: Morgan Kaufmann, 2002.

[17] G. Perlman. (2005, Feb.) Web-Based User Interface Evaluation with Questionnaires. [Online]. Available: http://www.acm.org/ perlman/question.html

[18] R. Pastor, J. Sánchez, and S. Dormido, "RELATED: A framework to publish Web-based laboratory control systems," in Preprints of the IFAC Workshop on Internet Based Control Education, Madrid, Spain, 2001, pp. 207-212.

[19] L. Dusseault, WebDAV: Next-Generation Collaborative Web Authoring. Englewood Cliffs, NJ: Prentice-Hall, 2004. 
Denis Gillet (S'87-M'88) received the Diploma degree in electrical engineering and the Ph.D. degree in control systems from the Ecole Polytechnique Fédérale de Lausanne (EPFL), Lausanne, Switzerland, in 1988 and 1995, respectively.

$\mathrm{He}$ is currently MER (Associate Professor) at EPFL. His research interests include optimal and hierarchical control systems, distributed e-learning systems, and sustainable interaction and real-time Internet services.

Dr. Gillet received the 2001 Recognition Award for Innovations and Accomplishments in Distance and Flexible Learning Methodologies for Engineering Education from the International Network for Engineering Education and Research (iNEER).

Anh Vu Nguyen Ngoc received the B.Sc. degree in computer science from Vietnam National University, Ho Chi Minh City, in 1997 and the Doctoral School degree in communication systems from the Ecole Polytechnique Fédérale de Lausanne (EPFL), Lausanne, Switzerland, in 2001. He is currently working toward the Ph.D. degree at the School of Engineering, EPFL.

Since August 2001, he has been a Research Assistant with the School of Engineering, EPFL. His research interests include e-learning, collaboration and interaction systems, computer-supported cooperative work (CSCW), human-computer interaction (HCI), and Web engineering.

Mr. Nguyen Ngoc received an Outstanding Paper Award from the ED-MEDIA conference in 2004.
Yassin Rekik received the Ph.D. degree in computer science from the Ecole Polytechnique Fédérale de Lausanne (EPFL), Lausanne, Switzerland, in 2001. He is a Senior Research Associate at EPFL and Professor at the University of Applied Sciences Neuchâtel, Switzerland. His research interests include Webbased learning, mobile learning, collaborative and group-oriented learning, and online experimentation and laboratory activities.

Dr. Rekik is currently involved in several national and international initiatives and projects, in particular, the European Network of Excellence ProLearn. 